\title{
A mathematical description of glitches in neutron stars
}

\author{
Maria Stella Mongiovì,${ }^{1 \star}$ Francesco G. Russo ${ }^{2 \star}$ and Michele Sciacca ${ }^{3,4 \star}$ \\ ${ }^{1}$ Dipartimento di Innovazione Industriale e Digitale, Università degli Studi di Palermo, Viale Delle Scienze, Edificio 8 , I-90128 Palermo, Italy \\ ${ }^{2}$ Department of Mathematics and Applied Mathematics, University of Cape Town, Private Bag X1, 7701, Rondebosch, Cape Town, South Africa \\ ${ }^{3}$ Dipartimento di Scienze Agrarie e Forestali, Università degli Studi di Palermo, Viale Delle Scienze, Edificio 4, I-90128 Palermo, Italy \\ ${ }^{4}$ Istituto Nazionale di Alta Matematica, Piazzale Aldo Moro, I-00185 Roma, Italy
}

Accepted 2017 March 31. Received 2017 March 24; in original form 2016 June 30

\begin{abstract}
In a pulsar, there are gaps and difficulties in our knowledge of glitches, mainly because of the absence of information about the physics of the matter of the star. This has motivated several authors to suggest dynamical models that interpret most of the astronomical data. Many predictions are based on the assumption that the inner part is analogous to the structure of matter of superfluids. Here, we illustrate a new mathematical model, partially inspired by the dynamics of superfluid helium. We obtain two evolution equations for the angular velocities (of the crust and of superfluid), which are supported by another evolution equation for the average vortex line length per unit volume. This third equation is more delicate from an analytical perspective and is probably at the origin of glitches. We identify two stationary solutions, corresponding to the straight vortex regime and the turbulent regime.
\end{abstract}

Key words: turbulence - pulsars: general-stars: neutron-stars: rotation.

\section{INTRODUCTION}

Neutron stars are compact self-gravitating objects, which are one of possible end products of stellar evolution. Their structure is fundamentally unknown. From physical and astronomical considerations, it is widely accepted that there are several distinct layers, which rotate at different angular velocities. The mass of a neutron star is about one solar mass $\left(\sim 10^{30} \mathrm{~kg}\right)$, but its radius is $\sim 10 \mathrm{~km}$, which is very small with respect to the size of the original star. At the top, we find the surface layer, which is composed of Fe nuclei and forms a closed-packed solid. The next layer, called the outer crust, contains a lattice of heavy nuclei and electrons. The inner crust is composed of a lattice of neutron-rich nuclei, free degenerate neutrons and degenerate electron gas. As soon as the density increases, the nuclei dissolve. The bulk of these stars consists essentially of a superfluid neutron liquid at the huge density of $\sim 10^{17} \mathrm{~kg} \mathrm{~m}^{-3}$ with a small concentration of protons and electrons, which may become superconductors, under certain circumstances. Finally, there may be an extremely compact core region, composed of hadrons, whose radius is actually unknown; for more details, see Packard (1972), Padmanabhan (2001), Andersson, Sidery \& Comer (2007), Fattoyev \& Piekarewicz (2010), Espinoza et al. (2011), Urama, Joshi \& Chukwude (2012) and Sedrakian, Hayrapetyan \& Baghdasaryan (2014).

Even if this picture of the star and the existence of superfluidity in neutron stars is not confirmed observationally, there are several aspects that allow us to suppose that the interiors of non-young ^E-mail: m.stella.mongiovi@unipa.it (MSM); francescog.russo@yahoo.it
(FGR); michele.sciacca@unipa.it (MS) pulsars may contain superfluid. For instance, the data of Page et al. (2011) and Shternin et al. (2011) are based on the study of the cooling process of a young pulsar (i.e. almost $330 \mathrm{yr}$ old), and the observational evidence of cooling of Cassiopeia A by Heinke \& Ho (2010) is interpreted as evidence of superfluidity in Page et al. (2011) and Shternin et al. (2011). However, superfluid models of neutron stars are not new in the literature, as evidenced by Migdal (1959), Baym, Pethick \& Pines (1969) and Baym, Bethe \& Pethick (1971). In fact, the presence of superfluid seems to describe better a series of phenomena, which were not noted in the so-called star quake models (see Padmanabhan 2001). The reader can refer to Haskell et al. (2015) and Howitt, Haskell \& Melatos (2016) for more information on the dynamical models that have been proposed in the study of pulsars.

When the neutron star originates, the conservation of the angular momentum leads to very rapid rotation. It is known that the average period of the neutron star increases very slowly at a constant rate (typically $\sim 10^{-9} \mathrm{~s} \mathrm{~d}^{-1}$ ), showing that the rotating neutron star is slowing down, and is gradually losing its rotational energy. The periods of neutron stars are remarkably stable once the systematic increase (as a result of radiation loss) is taken into account. Some pulsars may suddenly suffer small decreases of period, which are known as glitches. These jumps (in the rotational speeds) are phenomena of particular interest, which are noted in rotating superfluid neutron stars such as the Crab pulsar and the Vela pulsar. We mention Espinoza et al. (2011) and Wang et al. (2000), who describe the peculiarity of each pulsar and the stability of their periods. In particular, Espinoza et al. (2011) study the rotation of more than 700 pulsars and report significant data for glitches and periods of most of the pulsars that are known. Tables 1 and 2 of Espinoza et al. (2011) collect a series of information that is fundamental for the 
Table 1. Data for the Vela pulsar.

\begin{tabular}{lc}
\hline$M_{\text {tot }}$ & $1.2 \times\left(1.98855 \times 10^{30}\right) \mathrm{kg}$ \\
$R_{\text {tot }}$ & $10.5 \mathrm{~km}$ \\
$\tau$ & $950 \mathrm{~d}$ \\
$\tau_{1}$ & $30 \mathrm{~s}$ \\
$\Omega_{0}$ & $70 \mathrm{~s}^{-1}$ \\
$k_{\mathrm{r}}=k_{\mathrm{r}}^{\mathrm{c}}+k_{\mathrm{r}}^{\mathrm{s}}$ & $7.37 \times 10^{25} \mathrm{~J}$ \\
$I_{\mathrm{tot}}$ & $10^{38} \mathrm{~kg} \mathrm{~m}^{2}$ \\
$y_{\mathrm{av}}(1)-y_{\mathrm{c}}(1)$ & $20 \times 10^{-6}$ \\
\hline
\end{tabular}

Table 2. Data from Table 1 and data used in the plots.

\begin{tabular}{lc}
\hline$I_{\mathrm{c}}=10^{-1} I_{\text {tot }}$ & $10^{37} \mathrm{~kg} \mathrm{~m}^{2}$ \\
$I_{\mathrm{S}}=\left(1-10^{-1}\right) I_{\mathrm{tot}}$ & $9 \times 10^{37} \mathrm{~kg} \mathrm{~m}^{2}$ \\
$B=I_{\mathrm{c}} / I_{\text {tot }}$ & 0.1 \\
$A=I_{\mathrm{s}} / I_{\mathrm{tot}}$ & 0.9 \\
$k_{\mathrm{r}}^{\mathrm{c}}$ & $2.772 \times 10^{26}$ \\
$K_{\mathrm{c}}=\left(k_{\mathrm{r}}^{\mathrm{c}} / I_{\mathrm{c}}\right)\left(\tau / \Omega_{0}\right)$ & 0.0000325037 \\
$K_{\mathrm{s}}=\left(\left(k_{\mathrm{r}}-k_{\mathrm{r}}^{\mathrm{c}}\right) / I_{\mathrm{s}}\right)\left(\tau / \Omega_{0}\right)$ & $-2.65132 \times 10^{-6}$ \\
$a$ & 6.08001 \\
$b$ & 61.8001 \\
$D_{1}$ & 1 \\
$D_{2}$ & -0.0000173875 \\
\hline
\end{tabular}

present study of the periods of pulsars. Apart from the Vela pulsar, most neutron stars show irregular glitches with different sizes of magnitude and different temporal intervals between two consecutive glitches. However, the Vela pulsar is not the only pulsar that glitches at regular intervals with similar size (see Middleditch et al. 2006).

The paper is organized as follows. In Section 2, we provide a description of the dynamics of a neutron star and we provide a physical justification for the glitch. In Section 3, we illustrate the mathematical model, which is able to describe the phenomenology of the glitches in a neutron star. Then, in Section 4, we discuss two separate regimes: the straight vortex regime and the turbulent regime. Our model is applied to the Vela pulsar in Section 5, where we use MATHEMATICA to reproduce the glitches of the star. The results agree quite well with the existing literature. In Section 6, we explain our conclusions and new lines of investigation. Finally, in Appendix A, we report results on the turbulence in counterflowing rotating superfluid helium. Notations and terminology are standard and follow Donnelly (1991), Padmanabhan (2001), Jou \& Mongiovì (2004), Peralta et al. (2006, 2008), Melatos \& Peralta (2007) and Mongiovì \& Jou (2007).

\section{PHYSICAL PICTURE OF THE GLITCH}

Let us assume that a neutron star is made of two components, which rotate with different angular velocities and interact with each other: the superfluid neutron liquid, which corresponds to the superfluid component in the two-fluid model of superfluid helium, and the crust, which, in our view, also includes protons and electrons spread out inside the star (i.e. the so-called normal component). This is a simplified scenario, but, as we will see, it is able to describe the main features of the phenomenon. We refer to Haskell et al. (2015) for a detailed compendium of the mathematical models that have been proposed to describe the behaviour of pulsars. Here, it is impossible to summarize the efforts made over many decades to understand the astronomical features of these stars, but it is possible to definitively recognize a first set of models, based on the fact that the outer crust forms a crystalline crust that can support stress. This approach has justified glitches as a consequence of star quakes, but recent data such as the data of Espinoza et al. (2011), Page et al. (2011) and Shternin et al. (2011) - seem to support a second set of models, based on the presence of superfluid. Our model follows such a line of research.

According to our view, glitches are caused by the relative velocity between the two components (crust and superfluid) and the presence of quantized vortices. In neutron stars, a solid shell contains a superfluid neutron liquid, which cannot sustain rigid body rotation. Thus, the rotating superfluid is penetrated by a large number of quantized vortex lines, as happens for superfluid helium in rotating vessels.

In fact, in rotating superfluids, it is known that some quantized vortices (with quantized circulation) appear in the bulk for high enough angular velocity of the vessel (in He II, these vortices show a fixed core of $1 \AA$ and a quantum of circulation given by $\kappa=9.97 \times 10^{-4} \mathrm{~cm}^{2} \mathrm{~s}^{-1}$ ). We recall some information on the dynamics of superfluid helium in Appendix A, but the reader can find more details in Donnelly (1991), Barenghi, Donnelly \& Vinen (2001), Nemirovskii, Stamm \& Fiszdon (1993), Nemirovskii (2013) and Tsakadze \& Tsakadze (1980).

Following Donnelly (1991), the rotation sustains mainly straight quantized vortices, which (in stationary superfluid) must be parallel to the rotation vector and pinned to the crust of the star. However, various topologies of the vortex array are possible, and a transition between them can cause modifications of the angular momentum of the superfluid. This might be responsible for the glitches, as explained in more detail below.

A simplified sketch of the dynamics of a neutron star is summarized here.

\section{Pinned and almost straight vortex regime}

In rotating superfluid helium, it is known that the presence of straight quantized vortices causes the corotation of the normal and superfluid components (Donnelly 1991). In comparison with superfluid helium, the interior of the neutron star is also composed of these two components. We assume that the first component is protons and electrons in a normal state, which rotates rigidly with the crust; the second component, which is viscousless, does not detect friction apart from the presence of vortices. We must observe that, in a neutron star, pinned vortices are not strictly straight lines, as in a rotating cylinder filled with He II; in fact, they must be slightly curved near the crust and orthogonal to it. Nevertheless, for the sake of simplicity, in the following this state is equally called the straight vortex regime, in analogy with the rotating helium superfluid inside a cylinder (see Appendix A).

Let us suppose that both the crust and the inner part of the star corotate with an initial angular velocity $\Omega_{0}$, because of the presence of vortex filaments, which are (almost) parallel to the rotating axis and pinned to the crust (see Fig. 1).

The crust (and hence the normal component) slows down because of the loss of energy, some of which radiates away from the star and the remaining is supplied to the superfluid component, through the mutual interaction between the normal component (protons and electrons) and the vortex lines, which in some stars could cause a slow acceleration of the superfluid. In this scenario, vortex lines, which are strongly pinned to the crust, deform their straight configuration for two main reasons: (i) the crust slows down faster than the superfluid and the vortices move approximately with the same velocity as the superfluid, and as a consequence they deform and become longer; (ii) the mutual friction force between the two 


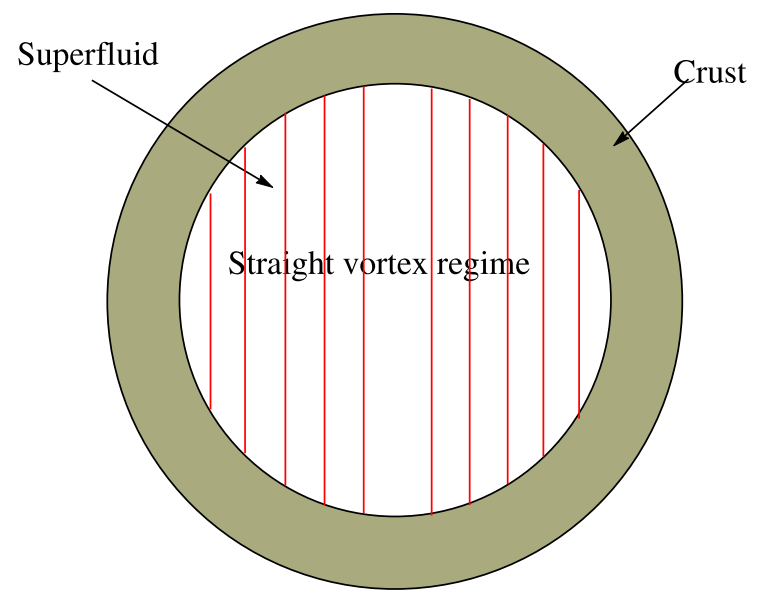

Figure 1. A schematic diagram of what occurs inside a neutron star in the straight vortex regime: the quantized vortices are (almost) straight lines, parallel to the rotating axis and pinned perpendicularly to the crust. In the diagram, only a few vortex lines are represented. Indeed, because of the very high angular velocity of the star, the density of the vortex lines is much higher.

components of the superfluid depends on the distance from the centre of the star and this means that the mutual friction force is not uniform all over the vortex filament. A further consequence is that the inter-vortex distance slows.

\section{Turbulent regime}

The slowing down of the crust increases the difference between the rotating velocities of the crust and superfluid, and hence the distribution of the mutual friction over the quantized vortices. It allows the formation of Kelvin waves over the vortices and the consequent appearance of the Donnelly-Glaberson instability (see Donnelly 1991). When the inter-vortex distance is of the same order as the amplitude of the waves, quantum turbulence appears and there is unpinning from the crust. It can be macroscopically referred to the difference between the two angular velocities of the crust and the inner part of the star. When it is higher than a critical value, the vortices can no longer keep an ordered configuration, and therefore they unpin catastrophically from the crust. After the unpinning, the vortices undergo reconnections and change their topology in the neutron star. Thus, a disordered tangle of quantized vortices is present in the interior of the star. This state is called the turbulent state, by analogy with the rotating turbulent superfluid helium subject to an external heat flow (see Appendix A and Figs 1 and 2). At the transition, this unpinning transfers angular momentum to the outer crust, which accelerates suddenly (i.e. there is a glitch); see Andersson et al. (2007), Melatos \& Peralta (2007) and Peralta et al. (2006, 2008). Thus, according to our model, glitches have two main causes: (i) the unpinning of the vortices from the crust, which makes the tension forces null (see Khalatnikov 1965; Jou, Mongiovì $\&$ Sciacca 2011); (ii) the sudden change of the distribution of the mutual friction force caused by the different topology of quantized vortices. The growth in the length of the vortex line density per unit volume and the near absence of tension force the crust and neutron superfluid to be matched in a very short time $\tau_{1}$ (i.e. at the end of this regime).

At the same time, the superfluid neutron quickly slows down until the difference between the two velocities of rotation is less than the critical value. This is a very rapid phenomenon; indeed, because of

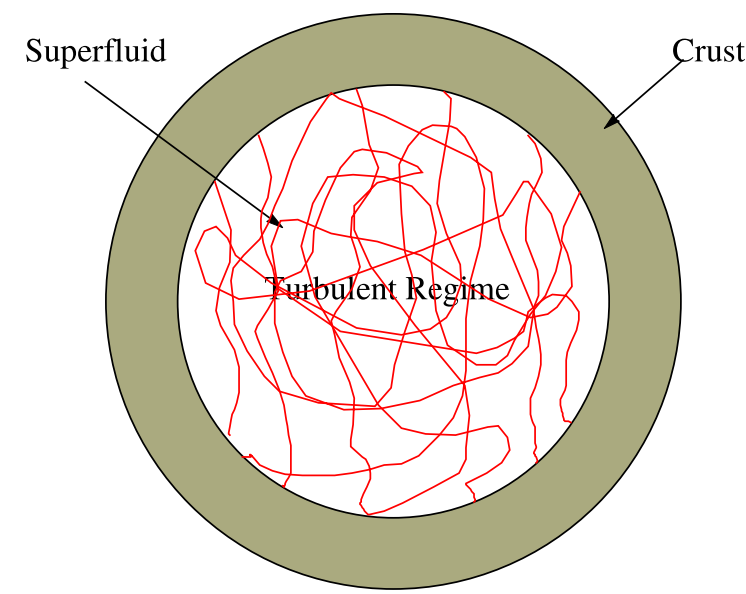

Figure 2. A schematic diagram of what occurs inside the neutron star in the turbulent regime (just after the glitch). Most of the quantized vortices are unpinned (the cause of the glitch) in such a way that the vortex tangle takes place inside the neutron star. As in Fig. 1, in the plot only a few vortex lines are represented.

the fast rotation, the vortex filaments tend to be parallel to the rotating axis, and therefore pin to the outer crust, and the two parts of the star (the outer crust and the inner part of the star) will corotate again.

As we have seen, we follow the standard view of pulsar glitches by assuming a superfluid component whose vortices are pinned to the crust, before the glitch. The superfluid cannot lose vorticity and spin down, lagging behind the normal fluid, which spins down along with the solid crust. We infer that the internal structure of any star contributes to the formation of glitches in different ways; that is, an inhomogeneous distribution of density and of normal and superfluid components contributes to the presence of inhomogeneous regions of pinning, or to regions with different local velocities and to local quantum turbulence. Thus, the microscopic view before any glitch is different from any other, and this would explain why glitches can vary in size over several orders of magnitude. Indeed, one glitch could be caused by the unpinning from some regions of the star, and the next glitch from other regions where a mild quantum turbulence has been kept.

This is probably the reason why in some neutron stars the size of glitches does not appear to depend on parameters such as the time since previous glitches. In fact, the straight vortex regime may become turbulent in principle at an arbitrary value of rotation. Following Alpar, Langer \& Sauls (1984) and the so-called 'snowplow model', we believe that this is a possible trigger mechanism, which is behind the nature of the glitches of Vela. As noted by Haskell et al. (2015, p. 24), this trigger mechanism can explain the glitch behaviour of Vela and the other quasi-periodic phenomena in nonyoung pulsars. However, it is in general surpassed by more advanced hypotheses such as the so-called 'vortex avalanches'. Because our aim is to explain and describe the formation of one glitch, we can focus our attention on the behaviour of the Vela pulsar.

\section{CONSTRUCTION OF THE MATHEMATICAL MODEL}

As we have said in Section 2, the outer layers and protons and electrons (inside the star) are called the crust, while the inner superfluid neutron liquid (inside the star) is called the superfluid.

In order to describe the dynamics of a glitch in a superfluid neutron star, we choose the average vortex line length per unit 
volume $L$ (i.e. the vortex line density) and the angular velocities $\Omega_{\mathrm{c}}$ and $\Omega_{\mathrm{s}}$ of the crust and the superfluid, respectively, as our fundamental variables. The variable $L$ provides information on the array of quantum vortex lines inside the neutron superfluid.

Let us assume that the dynamics of the star is governed by the equations

$$
\begin{aligned}
I_{\mathrm{c}} \frac{\mathrm{d} \Omega_{\mathrm{c}}}{\mathrm{d} t} & =-k_{\text {radiation }}^{\mathrm{c}}+K_{\text {friction }} \\
I_{\mathrm{s}} \frac{\mathrm{d} \Omega_{\mathrm{s}}}{\mathrm{d} t} & =-k_{\text {radiation }}^{\mathrm{s}}-K_{\text {friction }} \\
\frac{\mathrm{d} L}{\mathrm{~d} t} & =\left(\frac{\mathrm{d} L}{\mathrm{~d} t}\right)_{\text {formation }}-\left(\frac{\mathrm{d} L}{\mathrm{~d} t}\right)_{\text {destruction }},
\end{aligned}
$$

where $I_{\mathrm{c}}$ and $I_{\mathrm{s}}$ are the moments of inertia of the crust and superfluid, respectively. The quantities $k_{\text {radiation }}^{\mathrm{c}}=k_{\mathrm{r}}^{\mathrm{c}}$ and $k_{\text {radiation }}^{\mathrm{s}}=k_{\mathrm{r}}^{\mathrm{s}}$ determine the steady change in the rotational speed, due to the loss of rotational energy by radiation. Here, $K_{\text {friction }}$ is the torque due to the friction between the crust, the superfluid and the quantized vortex lines.

There are different contributions to $K_{\text {friction }}$; the more important contributions are the mutual friction force $\boldsymbol{F}_{\mathrm{MF}}$ and the vortex tension $\rho_{\mathrm{s}} \boldsymbol{T}$ (Donnelly 1991; Jou, Mongiovì \& Sciacca 2011). The first is a result of the interaction between superfluid vortices and the normal component (protons and electrons inside the star). The vortex tension force is a restoring force related to the curvature of the vortex lines, and its contribution is important near the pinning regions of vortex lines. These forces, when acting on vortices, can produce either a growth of line density $L$ or its decay, and this effect depends on the curvature of vortex lines.

We choose for $K_{\text {friction }}$ a linear dependence on $\Omega_{\mathrm{c}}$ and $L$ as it occurs in rotating He II. We then assume that the friction is almost absent for straight vortex lines, which occurs for $L=2 \Omega / \kappa$ (as happens in superfluid helium), where the crust and the superfluid corotate:

$K_{\text {friction }}=-\frac{1}{\tau} \frac{I_{\mathrm{s}} I_{\mathrm{c}}}{I_{\mathrm{s}}+I_{\mathrm{c}}}\left(\Omega_{\mathrm{c}}-\frac{\kappa L}{2}\right)$.

Here, $\tau$ is the characteristic time-scale of the coupling between the crust and the superfluid, and $\kappa=h / 2 m_{\mathrm{n}}$ is the quantum of vorticity (see Padmanabhan 2001), where $h$ is the Planck constant and $m_{\mathrm{n}}$ is the neutron mass, because the superfluidity in neutron stars originates from the formation of Cooper pairs (see Barenghi et al. 2001).

REMARK 1. The term $\kappa L / 2$ has the dimension $\mathrm{s}^{-1}$, which corresponds to the angular velocity of the vessel in rotating He II (see equation A2 in Appendix A), but here it coincides with $\Omega_{\mathrm{s}}=\Omega_{\mathrm{c}}$, only when the crust and superfluid corotate.

The evolution equation of $L$ is obtained by the difference between two terms, taking into account the formation and destruction of vortices (see Appendix A). Inspired by previous studies on superfluid helium by Mongiovì \& Jou (2007) and Sciacca, Mongiovì \& Jou (2008), we choose the decay term in the following form,

$$
\left(\frac{\mathrm{d} L}{\mathrm{~d} t}\right)_{\text {destruction }}=-\aleph \kappa L^{2},
$$

where $\boldsymbol{\aleph}$ is a dimensionless constant (see Vinen 1957a,b,c). We assume further that the growth of $L$ is due not only to $\Omega_{\mathrm{s}}$ but also to the relative angular velocity $\Omega_{\mathrm{sc}}=\Omega_{\mathrm{s}}-\Omega_{\mathrm{c}}$, which produces a situation inside the star that is analogous to that of the counterflow in rotating superfluid helium. In fact, Jou \& Mongiovì (2004) have shown that the rate of formation of vortices in $\mathrm{He}$ II in the counterflow in rotating containers depends on the counterflow velocity $V$ and on the intensity $\Omega$ of the angular velocity of the container (see Appendix A). In a neutron star, the role of $V$, which randomizes the vortex lines in the tangle, is substituted by $\Omega_{\text {sc }}$ and the role of $\Omega$, which tends to align vortex lines to the rotation axis, is substituted by $\Omega_{\mathrm{s}}$. Because we do not perform a microscopic derivation of the dynamics of $L$ in a neutron star, here we limit ourselves to deriving the evolution equation for $L$, using phenomenological and dimensionless ingredients. Dimensional analysis leads to the following expression for the vortex production:

$\left(\frac{\mathrm{d} L}{\mathrm{~d} t}\right)_{\text {formation }}=\kappa L^{2} \phi_{\mathrm{f}}\left(\frac{\Omega_{\mathrm{s}}}{k L}, \frac{\Omega_{\mathrm{sc}}}{\kappa L}\right)$.

For the function $\phi_{\mathrm{f}}$, we choose a quadratic dependence on its variables, obtaining

$$
\begin{aligned}
\left(\frac{\mathrm{d} L}{\mathrm{~d} t}\right)_{\text {formation }}= & \kappa L^{2}\left[\alpha_{1} \frac{\Omega_{\mathrm{sc}}}{k L}+\alpha_{2} \frac{\Omega_{\mathrm{s}}}{\kappa L}\right. \\
& \left.+\beta_{1}\left(\frac{\Omega_{\mathrm{s}}}{k L}\right)^{2}+\beta_{2} \frac{\Omega_{\mathrm{sc}} \Omega_{\mathrm{s}}}{(\kappa L)^{2}}\right],
\end{aligned}
$$

where $\alpha_{1}, \alpha_{2}, \beta_{1}$ and $\beta_{2}$ are dimensionless constants. The term $\left(\Omega_{\mathrm{sc}} / k L\right)^{2}$ has not been considered because of the physical idea according to which the crust and the inner part of the star rotate with angular velocities of the same order. This implies that the difference $\Omega_{\mathrm{sc}}=\Omega_{\mathrm{s}}-\Omega_{\mathrm{c}}$ is much smaller than $\Omega_{\mathrm{s}}$. This is also confirmed by the results in Section 5. Thus, the equation for $L$ becomes

$$
\frac{\mathrm{d} L}{\mathrm{~d} t}=-\aleph L^{2}+\left(\alpha_{1} \Omega_{\mathrm{sc}}+\alpha_{2} \Omega_{\mathrm{s}}\right) L+\frac{1}{\kappa}\left(\beta_{1} \Omega_{\mathrm{s}}^{2}+\beta_{2} \Omega_{\mathrm{sc}} \Omega_{\mathrm{s}}\right) .
$$

The stationary solutions of this equation are

$$
\begin{aligned}
L_{1,2}= & \frac{1}{2 \aleph \kappa}\left[\alpha_{1} \Omega_{\mathrm{sc}}+\alpha_{2} \Omega_{\mathrm{s}}\right. \\
& \left. \pm \sqrt{\left(\alpha_{1} \Omega_{\mathrm{sc}}+\alpha_{2} \Omega_{\mathrm{s}}\right)^{2}+4 \aleph\left(\beta_{1} \Omega_{\mathrm{s}}^{2}+\beta_{2} \Omega_{\mathrm{s}} \Omega_{\mathrm{sc}}\right)}\right] .
\end{aligned}
$$

Now, we choose $\alpha_{i}$ and $\beta_{i}$ in such a way that the two stationary solutions of equation (6) (assuming $\Omega_{\mathrm{c}}$ and $\Omega_{\mathrm{s}}$ constant) correspond to the two states described in Section 2. The first is for straight vortices, which depends only on the velocity of rotation $\Omega_{\mathrm{s}}$, and the second is for a disordered tangle of vortices, which depends on the combination of the two velocities $\Omega_{\mathrm{s}}$ and $\Omega_{\mathrm{sc}}$. Indeed, $\Omega_{\mathrm{s}}$ tends to align the vortex lines in the tangle, while $\Omega_{\mathrm{sc}}$ tends to randomize them (see Appendix A; Mongiovì \& Jou 2007; Jou \& Mongiovì 2004).

The assumption $L_{1}=2 \Omega_{\mathrm{s}} / \kappa$, mimicking a solid-body rotation, is due to the analogy with $\mathrm{He}$ II in a rotating container. If this happens, then the following conditions are obtained:

$\beta_{2}=-2 \alpha_{1}, \quad \beta_{1}=-2\left(\alpha_{2}-2 \aleph\right)$.

Note that here $\Omega_{\mathrm{s}}$ depends on $t$, because of the energy losses due to the radiation, while for He II in a rotating vessel $\Omega_{\mathrm{s}}$ is constant.

Thus, the solutions of equation (6) become

$L_{1}=\frac{2 \Omega_{\mathrm{s}}}{\kappa}$
$L_{2}=\frac{\alpha_{1}}{\aleph} \frac{\Omega_{\mathrm{sc}}}{\kappa}-\frac{\beta_{1}}{2 \aleph} \frac{\Omega_{\mathrm{s}}}{\kappa}$. 
Solution $L_{1}$ is independent of the relative angular velocity $\Omega_{\mathrm{sc}}$ and corresponds to the straight vortex regime, where almost rectilinear vortices are strongly pinned to the crust; in fact, vortex lines pin to the crust orthogonal to it, and therefore they can actually be straight lines only near the rotation axis of the star. Solution $L_{2}$ depends linearly on $\Omega_{\mathrm{sc}}$ and corresponds to the turbulent regime (i.e. vortices form a turbulent disordered tangle; see Fig. 2).

Thus, we obtain the system

$I_{\mathrm{c}} \frac{\mathrm{d} \Omega_{\mathrm{c}}}{\mathrm{d} t}=-k_{\mathrm{r}}^{\mathrm{c}}-\frac{1}{\tau} \frac{I_{\mathrm{s}} I_{\mathrm{c}}}{I_{\mathrm{s}}+I_{\mathrm{c}}}\left(\Omega_{\mathrm{c}}-\frac{\kappa L}{2}\right)$

$I_{\mathrm{s}} \frac{\mathrm{d} \Omega_{\mathrm{s}}}{\mathrm{d} t}=-k_{\mathrm{r}}^{\mathrm{s}}+\frac{1}{\tau} \frac{I_{\mathrm{s}} I_{\mathrm{c}}}{I_{\mathrm{s}}+I_{\mathrm{c}}}\left(\Omega_{\mathrm{c}}-\frac{\kappa L}{2}\right)$

$\frac{1}{\aleph \kappa} \frac{\mathrm{d} L}{\mathrm{~d} t}=-\left(L-\frac{2 \Omega_{\mathrm{s}}}{\kappa}\right)\left[L-\left(\frac{\alpha_{1}}{\aleph} \frac{\Omega_{\mathrm{sc}}}{\kappa}-\frac{\beta_{1}}{2 \aleph} \frac{\Omega_{\mathrm{s}}}{\kappa}\right)\right]$.

Finally, we obtain

$k_{\mathrm{r}}^{\mathrm{c}}+k_{\mathrm{r}}^{\mathrm{s}}=k_{\mathrm{r}}$,

where $k_{\mathrm{r}}$ is the total torque.

REMARK 2. Note that the loss of energy of the star is the result of two different contributions: the first is the electromagnetic radiation, and the second is the loss of energy at the smallest scales in the vortex lines. In fact, this occurs in the quantized vortices in superfluid helium (see Vinen 2001; Kozik \& Svistunov 2009).

REMARK 3. We consider the sign of the coefficients in equation (6). In fact, at the beginning of the turbulent regime, straight vortices are still pinned and $\Omega_{\mathrm{sc}}$ reaches its maximum value. Here, the angular velocity of the crust begins to grow, while that of the superfluid is decreasing (with respect to the average velocity of the whole star). This behaviour is because the inner part of the star transfers energy and angular momentum to the crust. Consequently, $K_{\text {friction }}$ must have a different sign in the two regimes. Furthermore, at the final time of the turbulent regime, we have assumed that the crust and superfluid corotate again, and therefore $\Omega_{\mathrm{sc}}=0$. This is reflected by $\beta_{1}$ being negative in equation (9).

\subsection{Dimensionless form}

To perform a mathematical study of this system, first we write the system in dimensionless form, introducing the following variables:

$y_{\mathrm{s}}=\frac{\Omega_{\mathrm{s}}}{\Omega_{0}}, \quad y_{\mathrm{c}}=\frac{\Omega_{\mathrm{c}}}{\Omega_{0}}, \quad z=\frac{\kappa L}{2 \Omega_{0}}, \quad q=\frac{t}{\tau}$.

Here, $\Omega_{0}$ is the characteristic angular velocity (note that it is the angular velocity of the crust and of the core, before the spin-down begins). Now, equation (10) can be rewritten as

$$
\begin{aligned}
\frac{\mathrm{d} y_{\mathrm{c}}}{\mathrm{d} q} & =-K_{\mathrm{c}}-A\left(y_{\mathrm{c}}-z\right) \\
\frac{\mathrm{d} y_{\mathrm{s}}}{\mathrm{d} q} & =-K_{\mathrm{s}}+B\left(y_{\mathrm{c}}-z\right) \\
\gamma \frac{\mathrm{d} z}{\mathrm{~d} q} & =-\left(z-y_{\mathrm{s}}\right)\left(z+a y_{\mathrm{sc}}-b y_{\mathrm{s}}\right),
\end{aligned}
$$

where

$$
y_{\mathrm{sc}}=y_{\mathrm{s}}-y_{\mathrm{c}}, \quad \gamma=\frac{1}{2 \aleph \tau \Omega_{0}}
$$

and we denote

$$
K_{\mathrm{c}}=\frac{k_{\mathrm{r}}^{\mathrm{c}} \tau}{I_{\mathrm{c}} \Omega_{0}}, \quad K_{\mathrm{s}}=\frac{k_{\mathrm{r}}^{\mathrm{s}} \tau}{I_{\mathrm{s}} \Omega_{0}}, \quad A=\frac{I_{\mathrm{s}}}{I_{\mathrm{s}}+I_{\mathrm{c}}},
$$

$$
B=\frac{I_{\mathrm{c}}}{I_{\mathrm{S}}+I_{\mathrm{c}}}, \quad a=-\frac{\alpha_{1}}{2 \aleph}, \quad b=-\frac{\beta_{1}}{4 \aleph} .
$$

Note that $\gamma$ can be written as

$\gamma=\frac{1}{\aleph \tau} \frac{L_{0}^{-1 / 2}}{\kappa L_{0}^{1 / 2}}=\frac{\tau^{\prime}}{\tau}=\frac{1}{\tau} \frac{\delta}{v_{l}}$,

where $\tau^{\prime}=\delta / v_{l}$ is the ratio between the initial inter-vortex space $\delta=L^{-1 / 2}$ and the typical turbulent superfluid velocity around a vortex $v_{l}=\aleph \kappa L^{1 / 2}$ (Sciacca et al. 2010).

In this paper, we assume that $\gamma \ll 1$; that is, the characteristic time of the dynamics of vortices $\left(\tau^{\prime}\right)$ is smaller than the characteristic time of coupling between the crust and superfluid $(\tau)$. In terms of the observational data, this is what happens in a pulsar with good approximation. Indeed, a rough evaluation of $\tau^{\prime}$ can be achieved by assuming that the constant $\aleph$ is of the order of the unit, as it occurs in superfluid helium. In the straight vortex regime, $\tau^{\prime} \simeq(\kappa L)^{-1} \equiv$ $\left(2 \Omega_{\mathrm{s}}\right)^{-1}$. Because $\Omega_{\mathrm{s}}$ is of the same order of magnitude as $\Omega_{0}$, so $\tau^{\prime} \simeq 0.007 \mathrm{~s}$ for $\Omega_{0}=68 \mathrm{~s}^{-1}$. This implies that the characteristic time $\tau^{\prime}$ turns out to be smaller than $\tau$ (in principle, this implies that the dynamics of $L$ is much faster than the dynamics of the angular velocities of the crust and of the inner part of the star).

\section{DYNAMICAL BEHAVIOUR OF THE NEUTRON STAR}

Under the hypothesis that the coefficient $\gamma$ is negligible, the third equation of system (13) provides two steady solutions for $z$

$$
\begin{aligned}
& z_{1}=y_{\mathrm{s}} \\
& z_{2}=-a y_{\mathrm{sc}}+b y_{\mathrm{s}},
\end{aligned}
$$

which correspond to the values in equation (9) for $L$. The first solution $z_{1}$ corresponds to the straight vortex regime (i.e. almost rectilinear pinned vortices), whereas $z_{2}$ corresponds to the turbulent regime (i.e. a disordered tangle of vortices).

In the following, we study the solutions of the system (13) separately in the two different regimes: straight vortex $\left(z=z_{1}\right)$ and turbulent $\left(z=z_{2}\right)$.

\subsection{State 1: straight vortex regime}

Let us assume that initially the inner part of the star and the outer crust corotate in such a way that $y_{\mathrm{c}}=y_{\mathrm{s}}=1$ (i.e. $\Omega_{\mathrm{c}}=\Omega_{\mathrm{s}}=\Omega_{0}$ ). Here, the rigid-body rotation is going to happen.

In the case described here, the vortex line density $z$ is steady; $y_{\mathrm{c}}$ and $y_{\mathrm{s}}$ (which we denote by $y_{\mathrm{c}}^{(1)}$ and $y_{\mathrm{s}}^{(1)}$, in order to have a suitable notation with the corresponding solutions of Section 4.2) change in agreement with equation (13). In fact, the system (13) becomes:

$$
\begin{aligned}
\frac{\mathrm{d} y_{\mathrm{c}}^{(1)}}{\mathrm{d} q} & =-K_{\mathrm{c}}-A\left[y_{\mathrm{c}}^{(1)}-z\right] \\
\frac{\mathrm{d} y_{\mathrm{s}}^{(1)}}{\mathrm{d} q} & =-K_{\mathrm{s}}+B\left[y_{\mathrm{c}}^{(1)}-z\right] \\
z & =y_{\mathrm{s}} .
\end{aligned}
$$

Substituting $z=z_{1}=y_{\mathrm{s}}^{(1)}$ in the first two equations, the following simplified system is obtained,

$$
\begin{aligned}
& \frac{\mathrm{d} y_{\mathrm{c}}^{(1)}}{\mathrm{d} q}=-K_{\mathrm{c}}-A\left[y_{\mathrm{c}}^{(1)}-y_{\mathrm{s}}^{(1)}\right] \\
& \frac{\mathrm{d} y_{\mathrm{s}}^{(1)}}{\mathrm{d} q}=-K_{\mathrm{s}}+B\left[y_{\mathrm{c}}^{(1)}-y_{\mathrm{s}}^{(1)}\right]
\end{aligned}
$$


whose solutions, which satisfy the initial conditions $y_{\mathrm{c}}^{(1)}(0)=$ $y_{\mathrm{s}}^{(1)}(0)=1$, are

$y_{\mathrm{c}}^{(1)}(q)=y_{\mathrm{av}}(q)-A\left(K_{\mathrm{c}}-K_{\mathrm{s}}\right)\left(1-e^{-q}\right)$

$y_{\mathrm{s}}^{(1)}(q)=y_{\mathrm{av}}(q)+B\left(K_{\mathrm{c}}-K_{\mathrm{s}}\right)\left(1-e^{-q}\right)$,

Here, we have defined the dimensionless average angular velocity

$y_{\mathrm{av}}(q):=A y_{\mathrm{s}}+B y_{\mathrm{c}}=1-\left(A K_{\mathrm{s}}+B K_{\mathrm{c}}\right) q$.

Equations (18) correspond to the following solutions for $\Omega_{\mathrm{c}}$ and $\Omega_{\mathrm{s}}$ :

$\Omega_{\mathrm{c}}^{(1)}(t)=\Omega_{\mathrm{av}}(t)-A\left(K_{\mathrm{c}}-K_{\mathrm{s}}\right)\left(1-e^{-t / \tau}\right)$

$\Omega_{\mathrm{s}}^{(1)}(t)=\Omega_{\mathrm{av}}(t)+B\left(K_{\mathrm{c}}-K_{\mathrm{s}}\right)\left(1-e^{-t / \tau}\right)$.

Equation (19) in dimension form provides the average angular velocity of the star (which loses energy because of the electromagnetic radiation and the sound emission at the lowest scales). This is essentially the moment of inertia-weighted average of the angular velocities of the two components:

$\Omega_{\mathrm{av}}=\frac{I_{\mathrm{s}}}{I_{\mathrm{s}}+I_{\mathrm{c}}} \Omega_{\mathrm{s}}+\frac{I_{\mathrm{c}}}{I_{\mathrm{s}}+I_{\mathrm{c}}} \Omega_{\mathrm{c}}$.

At time $t_{0}=0$, they corotate so we have

$\Omega_{\mathrm{av}}(t)=\Omega_{0}-\frac{k_{\mathrm{r}}^{\mathrm{s}}+k_{\mathrm{r}}^{\mathrm{c}}}{I_{\mathrm{s}}+I_{\mathrm{c}}} t=\Omega_{0}-\frac{k_{\mathrm{r}}}{I_{\mathrm{s}}+I_{\mathrm{c}}} t$.

The difference between the two solutions (18) is

$y_{\mathrm{sc}}^{(1)}=\left(K_{\mathrm{c}}-K_{\mathrm{s}}\right)\left(1-e^{-q}\right)$.

This provides the relative angular velocity of the inner part of the star with respect to the crust:

$\Omega_{\mathrm{sc}}^{(1)}(t)=\tau\left(\frac{k_{\mathrm{r}}^{\mathrm{c}}}{I_{\mathrm{c}}}-\frac{k_{\mathrm{r}}^{\mathrm{s}}}{I_{\mathrm{s}}}\right)\left[1-e^{-(t / \tau)}\right]$.

Of course, if $K_{\mathrm{s}}=K_{\mathrm{c}}$, then $\Omega_{\mathrm{sc}}=0$ for all $t$. This reflects the fact that the crust and the superfluid always rotate with the same velocities. This allows us to assume $K_{\mathrm{s}} \neq K_{\mathrm{c}}$. The case $K_{\mathrm{s}}=0$ has been already studied by Mongiovì \& Sciacca (2010).

Note also that $K_{\mathrm{c}}>K_{\mathrm{s}}$ because the relative velocity $\Omega_{\mathrm{sc}}$ has to be positive. In fact, the velocity of the crust is lower than the velocity of the inner part of the star, which rotates together with the straight vortices.

The relative angular velocity $\Omega_{\mathrm{sc}}$ in equation (23) asymptotically tends to the value $K_{\mathrm{c}}-K_{\mathrm{s}}$. The sudden appearance of glitches, caused by the unpinning of the vortices from the crust, confines the growth of $\Omega_{\mathrm{sc}}$ to the value (23) with $t=\tau$. We can compute some parameters of a given star, once we know from astronomic observations the time $\tau$ and the angular velocity of the star just after the glitch. Indeed, if $t=\tau$, then $q=1$, and so we obtain

$y_{\mathrm{sc}}^{(1)}(1)=\left(K_{\mathrm{c}}-K_{\mathrm{s}}\right)\left(1-e^{-1}\right)$.

After the time $\tau$ (i.e. for $q=1$ ), the difference $y_{\mathrm{sc}}$ between the two angular velocities reaches its critical value $y_{\mathrm{sc}}^{\text {crit }}$, and the quantized vortices unpin from the crust, transferring some momentum to the crust. This is the origin of the glitch. Indeed, we believe that the unpinning of the straight vortex filaments from the crust (caused by the Donnelly-Glaberson instability) strongly reduces the tension between the vortices and the crust, and changes the topology of the vortex array. This sudden event allows the crust to rotate faster because of the small tension of quantized vortices. This trivial scenario depends strongly on the complexity of the interior of the star as well as of the crust. Indeed, local inhomogeneities in the interior of the star can encourage the formation of regions with a higher concentration of vortex filaments, or regions with a higher remnant of straight vortex filaments, even after a previous glitch. Hence, the two consecutive glitches may be caused by two different internal regions of the star. Furthermore, the complexity of the crust with some star quakes could make the appearance of glitches easier, as well as the amount of protons and electrons (which are in a normal state) and of the superfluid component in the interior of the star. All these ingredients make glitches more frequent in time and varying in size. However, our aim is to describe the behaviour between two consecutive glitches. For this reason, we have considered the dimensionless form of our dynamical equations, which abstracts from the particular glitch considered. We claim that our model is able to describe most of the glitches reported in Espinoza et al. (2011), even though the unpinning of the vortices is artificially inserted in our model.

\subsection{State 2: turbulent regime}

If $z=z_{2}$, then the system (10) becomes

$\begin{aligned} \frac{\mathrm{d} y_{\mathrm{c}}^{(2)}}{\mathrm{d} q} & =-K_{\mathrm{c}}-A\left[y_{\mathrm{c}}^{(2)}-z_{2}\right] \\ \frac{\mathrm{d} y_{\mathrm{s}}^{(2)}}{\mathrm{d} q} & =-K_{\mathrm{s}}+B\left[y_{\mathrm{c}}^{(2)}-z_{2}\right] \\ z_{2} & =-a y_{\mathrm{sc}}^{(2)}+b y_{\mathrm{s}}^{(2)},\end{aligned}$

where we use the notation $y_{\mathrm{c}}^{(2)}$ and $y_{\mathrm{s}}^{(2)}$ in order to avoid ambiguities with the previous case in Section 4.1. We obtain

$\frac{\mathrm{d} y_{\mathrm{c}}^{(2)}}{\mathrm{d} q}=-K_{\mathrm{c}}-A\left[(1-a) y_{\mathrm{c}}^{(2)}+(a-b) y_{\mathrm{s}}^{(2)}\right]$

$\frac{\mathrm{d} y_{\mathrm{s}}^{(2)}}{\mathrm{d} q}=-K_{\mathrm{s}}+B\left[(1-a) y_{\mathrm{c}}^{(2)}+(a-b) y_{\mathrm{s}}^{(2)}\right]$.

Putting $D_{1}=A(1-a)+B(b-a)$ and $D_{2}=K_{\mathrm{c}}(1-a)-K_{\mathrm{s}}(b-a)$, we find the solutions

$y_{\mathrm{c}}^{(2)}=\frac{A}{D_{1}} C \exp \left(-D_{1} q\right)+\frac{b-a}{D_{1}} y_{\mathrm{av}}-A \frac{D_{2}}{D_{1}^{2}}$

$y_{\mathrm{s}}^{(2)}=-\frac{B}{D_{1}} C \exp \left(-D_{1} q\right)+\frac{1-a}{D_{1}} y_{\mathrm{av}}+B \frac{D_{2}}{D_{1}^{2}}$,

where $C=(1-a) c_{1}+(a-b) c_{2}$ and

$y_{\mathrm{av}}=A y_{\mathrm{s}}+B y_{\mathrm{c}}=\left(A c_{2}+B c_{1}\right)-\left(B K_{\mathrm{c}}+A K_{\mathrm{s}}\right) q$,

which must be equal to equation (19). So we equalize and we find that

$A c_{2}+B c_{1}=1$.

In equation (27), we recognize the contributions of an exponential term - which contributes in a different way, depending on whether it is positive or negative - and of a linear term. The values $c_{1}$ and $c_{2}$ are constants of integration, which can be found by solving the Cauchy problem

$y_{\mathrm{c}}^{(2)}(1)=y_{\mathrm{c}}^{(1)}(1)$

$y_{\mathrm{s}}^{(2)}(1)=y_{\mathrm{s}}^{(1)}(1)$

that is, by imposing that the final values of State 1 coincide with those of State 2.

REMARK 4. Only two of the three conditions (29) and (30) are independent. From these, we can determine $c_{1}$ and $c_{2}$ through the other parameters (such as, for instance, $a$ and $b$ ). 


\subsection{Conditions on the coefficients}

The neutron star is in the straight vortex regime for $q \in[0,1]$ (or $t \in[0, \tau]$ ) and in the turbulent regime for $q \in\left[1,1+q_{1}\right]$ (or $t \in\left[\tau, \tau_{1}\right]$ ) with $q_{1}=\tau_{1} / \tau$, where $\tau_{1}$ is the duration of the glitch (i.e. how long the sudden acceleration of the crust lasts). For instance, astronomical data from the Vela pulsar show that the angular velocity of the crust undergoes a step of almost 20 part on $10^{6}$ at the glitch $(q=1)$ compared with the average angular velocity. Because our model assumes that, at $q=0$, the crust and superfluid corotate $\left(y_{\mathrm{s}}=y_{\mathrm{c}}=y_{\mathrm{av}}\right)$, we deduce that at the glitch $(q=1)$ this gap is

$y_{\mathrm{av}}(1)-y_{\mathrm{c}}(1)=A\left(K_{\mathrm{c}}-K_{\mathrm{s}}\right)\left(1-e^{-1}\right)$.

The formed gap runs out at $q=1+q_{1}=q_{2}$ (i.e. at the end of the turbulent regime). After this regime, the crust and the superfluid corotate again, and $y_{\mathrm{sc}}^{(2)}\left(q_{2}\right)=0$, and so we note the straight vortex regime again. Therefore, we have a further condition between the coefficients of the equations and the astronomical data:

$$
\begin{aligned}
y_{\mathrm{sc}}^{(2)}\left(q_{2}\right)= & \frac{1-b}{D_{1}} y_{\mathrm{av}} \\
& +\frac{D_{2}}{D_{1}^{2}}\left[1-\frac{D_{1} C}{D_{2}} \exp \left(-D_{1} q_{2}\right)\right]=0 .
\end{aligned}
$$

The physical meaning of this request comes from the quantum mechanical request that the microscopic superfluid component is curl-free and from the fact that the straight vortex regime is the most favourable configuration from an energetic point of view, in a rotating container (Donnelly 1991). Thus, the straight vortex regime is the final configuration towards which the quantum fluid inside the star points over time $\tau_{1}$. To establish this configuration entails the appearance of vortex tension with the crust and of a different topology of the vortex array. Thus, the sudden increase of the angular velocity of crust is a result of the appearance of quantum turbulence, and it finishes when quantum turbulence is again converted into the straight vortex regime, which requires rigid-body rotation as occurs in rotating superfluid helium (Donnelly 1991).

However, because of inhomogeneities in the interior of the star, in some glitches the straight vortex regime could not be the final configuration; however, an intermediate configuration of mild turbulence could be reached. This configuration would be a metastable configuration, and therefore other glitches would be produced, until the straight vortex regime is reached. This could explain why, in the youngest neutron stars, glitches have different frequencies and dimensions.

The previous condition (32) is valid under the assumption that the final configuration of the glitch is that of rectilinear vortices parallel to the rotation axis. After the turbulent regime, the inner part of the neutron star will again be in the straight vortex regime (i.e. in State 1), with the following Cauchy problem:

$y_{\mathrm{c}}^{(1)}\left(q_{2}\right)=y_{\mathrm{c}}^{(2)}\left(q_{2}\right)=1-\left(A K_{\mathrm{s}}+B K_{\mathrm{c}}\right) q_{2}$

$y_{\mathrm{s}}^{(1)}\left(q_{2}\right)=y_{\mathrm{s}}^{(2)}\left(q_{2}\right)=1-\left(A K_{\mathrm{s}}+B K_{\mathrm{c}}\right) q_{2}$.

REMARK 5. As in the previous case, only one of these conditions is independent.

Summarizing, from the knowledge of equation (31), the values of $K_{\mathrm{c}}$ and $K_{\mathrm{s}}$ follow, once the value of $A$ is known. The exact values of $A$ and $B$ (or $I_{\mathrm{s}}$ and $I_{\mathrm{c}}$ ) are essentially unknown in our simplified model, because we do not know the effective radius of the crust and of the inner part of the star. In the following, we choose $I_{\mathrm{s}}>I_{\mathrm{c}}$ as in Sedrakian, Hayrapetyan \& Sedrakian (2007) and Sedrakian et al. (2014), as this seems to be accepted in the existing literature.

From the conditions (29), (30) and (32) and the definitions of $D_{1}$ and $D_{2}$, the coefficients $c_{1}, c_{2}, a, b$ and $D_{2}$ can be easily expressed in terms of the coefficient $D_{1}$, which controls the growth of the exponential term in the solutions (27). All these coefficients are then known once all the parameters of the neutron stars are substituted.

\section{APPLICATION TO THE VELA PULSAR}

The aim of this section is to apply the model, which has been proposed and analysed in the previous sections, to a concrete example (i.e. the Vela pulsar), even though our results can be easily applied to any other pulsar. The main data of the Vela pulsar are reported in Table 1 and are taken from Dodson, Lewis \& McCulloch (2007), Yu et al. (2013) and Padmanabhan (2001). In Table 2, we report the values obtained from Table 1 and used in our model. The values of $c_{1}, c_{2}, a, b$ and $D_{2}$ are obtained using equations (29), (30) and (32) and the definitions of $D_{1}$ and $D_{2}$ below equation (26) for a suitable choice. We fixed $D_{1}=1$ for the sake of simplicity in the simulations. One of the main difficulties is to find the inertia momentum $I_{\mathrm{c}}$ and $I_{\mathrm{s}}$ because we do not know the exact regions of the star that belong to the crust and to the superfluid. Because we have checked that our model also works well with different relative choices of $I_{\mathrm{c}}$ and $I_{\mathrm{S}}$ with the condition that $I_{\mathrm{S}}>I_{\mathrm{c}}$, we use the inertia momentum $I_{\mathrm{c}}=10^{-1} I_{\mathrm{tot}}$ and hence $I_{\mathrm{s}}=I_{\mathrm{tot}}-I_{\mathrm{c}}=9 \times 10^{-1} I_{\mathrm{tot}}$. A more detailed discussion of this choice is made in Section 6. Regarding the other data reported in Table 1 , we use the definitions $A=I_{\mathrm{s}} / I_{\text {tot }}$ and $B=I_{\mathrm{c}} / I_{\text {tot }}$ for $A$ and $B$, respectively, and then equation (31) for yielding $k_{\mathrm{r}}$ and hence $K_{\mathrm{c}}$ and $K_{\mathrm{s}}$ from $K_{\mathrm{c}}=\left(k_{\mathrm{r}}^{\mathrm{c}} / I_{\mathrm{c}}\right)\left(\tau / \Omega_{0}\right)$ and $K_{\mathrm{s}}=\left(\left(k_{\mathrm{r}}-k_{\mathrm{r}}^{\mathrm{c}}\right) / I_{\mathrm{s}}\right)\left(\tau / \Omega_{0}\right)$.

We note that the values of Table 2 are connected with the choice of $I_{\mathrm{c}}, I_{\mathrm{s}}$ and $D_{1}$. Thus, different values of the duration of the glitch or of the momenta $I_{\mathrm{c}}$ and $I_{\mathrm{s}}$ can significatively influence the set of numerical values in Table 2. In particular, the lack of knowledge of the amount of vortex lines inside the star makes the determination of $a$ and $b$ variable. However, we point out that some sophisticated calculations are involved, in order to have all the parameters that are dependent on $D_{1}$ only. As shown in the figures, the model grasps the main features of the appearance of the glitch in the neutron star.

The positive values of $a$ and $b$ imply that $\alpha_{1}$ and $\beta_{1}$ are negative in equations (5) and (6). From equation (8), it follows that $\alpha_{2}$ and $\beta_{2}$ are positive (as $\aleph>0$ ). Physically, this means that the positive terms contribute to the growth of vortex line density while the negative terms obstruct it. The negative contribution of $\alpha_{1} \Omega_{\mathrm{sc}}$ stands for the transferring of the energy lost by the vortex tangle (and hence also from the inner part of the star) to the crust to raise its velocity. The high value of the coefficient $b$ means that there is a high amount of quantized vortex line density $z_{2}$ (or $L$ ) inside the neutron star in the turbulent regime, which reflects the loss of the rotational velocity of the superfluid during the glitch. These coefficients are strongly related to the coefficients of mutual friction, as shown by Jou, Mongiovì \& Sciacca (2011), which reflects the contribution of the mutual friction force to the appearance of the glitch, as also found in van Eysden \& Melatos (2010) and Howitt et al. (2016).

In Fig. 3, the two dimensionless velocities of the crust and of the inner part of the neutron star, $y_{\mathrm{c}}$ and $y_{\mathrm{s}}$, respectively, are plotted both in the straight vortex regime and in the turbulent regime. Here we assume (for the sake of illustration) that duration and frequency (after the glitch occurs again) are constant, but it must be noted that the glitches are in general neither periodic nor with the same intensity. In Fig. 3, we have drawn the velocities among three consecutive 


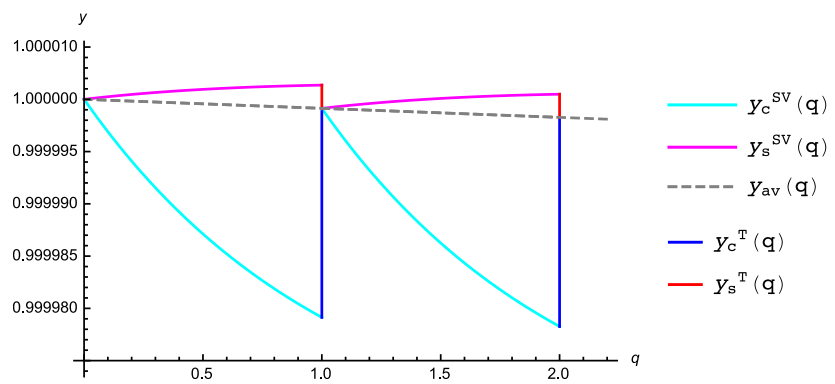

Figure 3. Plots of the velocity of the crust $y_{\mathrm{c}}(q)$ and of the superfluid $y_{\mathrm{S}}(q)$, in both the straight vortex regime (SV) and the turbulent regime (T). The jumps of the velocities reflect the glitches, which last for a very short time. We have chosen the value reported in Table $1 \tau_{1}=30 \mathrm{~s}$ or $q_{1}=3.65497 \times 10^{-7}$.

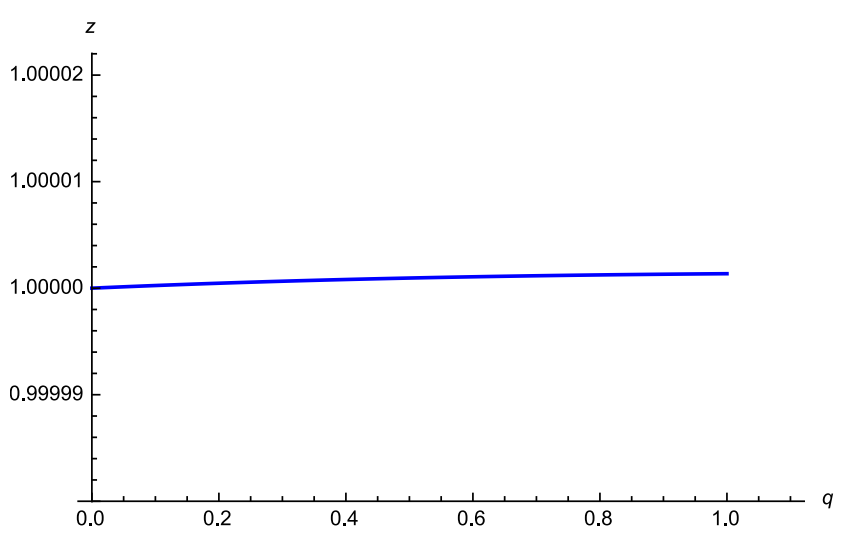

Figure 4. Vortex line density in the straight vortex regime.

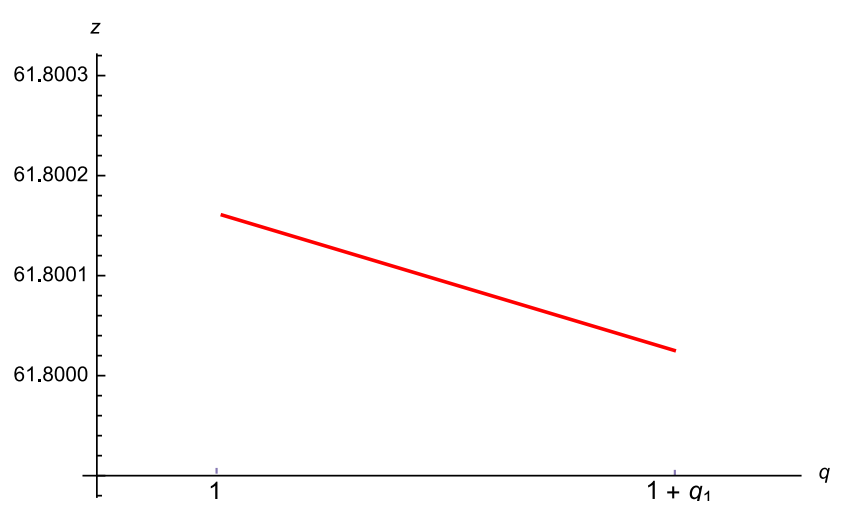

Figure 5. Vortex line density in the turbulent regime (glitch).

glitches. As shown by Fig. 3, it is hard to appreciate the duration of the glitches in these plots because it lasts only $q_{1}=3.65497 \times 10^{-7}$ ( $\left.\tau_{1}=30 \mathrm{~s}\right)$ compared to $q=1(\tau=950 \mathrm{~d})$. This is why we have devoted Fig. 6 to a more detailed analysis of this circumstance. In Figs 4 and 5, the dimensionless vortex line density is plotted in the straight vortex regime and in the turbulent regime, respectively. Note that in Fig. 4 the amount of vortex line grows, because of the appearance of Kelvin waves along the straight vortices, induced by the growth of $\Omega_{\mathrm{sc}}$ (a similar phenomenon has been studied by Brugarino, Mongiovì \& Sciacca 2015) for He II.

At $q=1$, the straight vortex regime ceases, because the topology of the vortex lines changes: the vortex tangle takes part in the superfluid, and the vortex line density undergoes a sudden increase

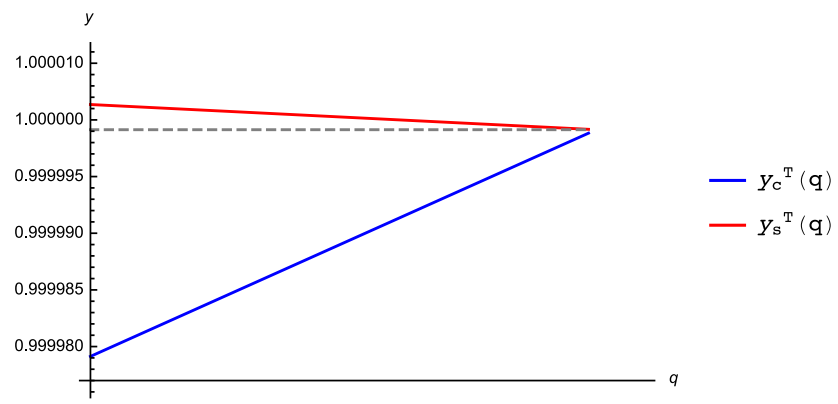

Figure 6. An enlargement of the plots shown in Fig. 3 to show the jump in the two velocities (glitch).

at $q=1$, as shown in Fig. 6. Consequently, the angular superfluid velocity decreases and the velocity of the crust increases, as shown in Fig. 6, until the two velocities coincide again at $q=q_{2}$ and the straight vortex regime is restored.

\section{CONCLUSIONS}

In this paper, we have proposed a simplified mathematical model to describe glitches in pulsars. We have found justifications in the quantized vortex lines that fill the superfluid neutron liquid, combined with the slowing down of the crust. This phenomenon is mainly related to the outer crust, which allows a rate of growth of the relative velocity between the crust and the superfluid. Two main regimes occur: a straight vortex regime and a turbulent regime. The crust and the superfluid corotate in such a way that vortex lines arrange like straight lines parallel to the rotation axis and pinned to the outer crust. Because of the slowing down of the crust, the relative velocity increases and acts on the vortices both by means of the mutual friction force (inside the star) and by stretching the vortices in the pinning regions (outer crust). When the amplitude of the Kelvin waves reaches the inter-vortex distance between consecutive vortices or the tension in the pinning regions becomes strong enough, the topology of the vortices (inside the star) changes, and a disordered vortex tangle originates. The sudden unpinning of the vortices and the decrease of the vortex tension supply momentum to the crust at the cost of the momentum of the superfluid. This change of the dynamical system produces a sudden spin-up of the angular velocity of the crust, which is in fact the glitch.

We modelled the above scenario using the system of equations (10) (or the dimensionless form (13)), involving the vortex line density $L$, the velocity of the crust $\Omega_{\mathrm{c}}$ and the velocity of the inner part of the star (superfluid) $\Omega_{\mathrm{s}}$. The evaluation made at the end of Section 2 allows us to assume that $\gamma=\tau^{\prime} / \tau \ll 1$ (i.e. the characteristic time $\tau^{\prime}$ for the dynamics of the vortex line is small with respect to $\tau$ ). Two stationary solutions $L=L_{1}$ and $L=L_{2}$ for the third equation of system (10) have been found, which then model the straight vortex regime and the turbulent regime, respectively.

We have made the following requirements:

(i) the velocity of the whole star $\Omega_{\mathrm{av}}$ decreases with the same rule in both regimes;

(ii) there is continuity of the solution $\Omega_{\mathrm{s}}$ and $\Omega_{\mathrm{c}}$ in the transition between two consecutive regimes;

(iii) the difference between the two velocities, $\Omega_{\mathrm{s}}$ and $\Omega_{\mathrm{c}}$, increases in the straight vortex regime, but decreases in the turbulent regime;

(iv) the value of $L$ increases at $t=\tau$. 
The above requirements have allowed us to find the values of some parameters of the model. We have a certain freedom for the choice of $I_{\mathrm{S}}$ and $I_{\mathrm{c}}$, and our model works well with the condition that $I_{\mathrm{S}}>I_{\mathrm{c}}$. These requirements differ slightly from those in Sedrakian et al. (2007, 2014) (see Table 2), because they are adapted to our simulations. More precisely, Fig. 3 plots the dimensionless angular velocities of the superfluid $y_{\mathrm{s}}$ and the crust $y_{\mathrm{c}}$, in the straight vortex regime and turbulent regime. This figure also reproduces the dynamics between three consecutive glitches (we have assumed that the gap between two consecutive glitches is constant). In the straight vortex regime, the velocity of the crust suffers a greater decrease with respect to the superfluid velocity. The situation suddenly changes when the glitch takes place, as shown by Fig. 6, in such a way that the two velocities take the same value again after a time $\tau_{1}$ (or $\left.q_{2}\right)$.

We have assumed, for the sake of simplicity, that $I_{\mathrm{s}}=9 I_{\mathrm{c}}$. We stress that the main behaviour shown in all the figures does not change significantly by changing the coefficient $D_{1}$. We have indeed seen that by changing coefficient $D_{1}$, all the coefficients $c_{1}, c_{2}, D_{2}$ and $a$ change apart from $b$, which remains almost constant. This implies that $z$ is not as affected by $D_{1}$. This is not the case when one changes the values of $I_{\mathrm{S}}$ and $I_{\mathrm{c}}$. More efforts will be made in future studies. In fact, we plan to generalize the above model in the following directions:

(i) application of our model to different pulsars;

(ii) additional study in presence of polarization and vortex diffusion (Saluto, Jou \& Mongiovì 2014a,b);

(iii) derivation of equations of a local nature.

\section{ACKNOWLEDGEMENTS}

The authors acknowledge the anonymous referee for suggestions and comments. MS and MSM acknowledge financial support from the National Group of Mathematical Physics (GNFM-INDAM) of Italy. FGR thanks the University of Cape Town for Grant URC/459235 and the Università di Palermo for the project 'Modelli Matematici di Sistemi Complessi e Studio di Fenomeni di Trasporto' with ref. no. 2551/2012.

\section{REFERENCES}

Alpar M. A., Langer S. A., Sauls J. A., 1984, ApJ, 282, 533

Andersson N., Sidery T., Comer G. L., 2007, MNRAS, 381, 747

Barenghi C. F., Donnelly R. J., Vinen W. F., 2001, Quantized Vortex Dynamics and Superfluid Turbulence. Springer, Berlin

Baym G., Pethick C. J., Pines D., 1969, Nature, 224, 673

Baym G., Bethe H. A., Pethick C. J., 1971, Nucl. Phys. A, 175, 225

Brugarino T., Mongiovì M. S., Sciacca M., 2015, Zeitschrift für Angewandte Mathematik und Physik, 66, 1081

Dodson R., Lewis D., McCulloch P., 2007, Ap\&SS, 308, 585

Donnelly R. J., 1991, Quantized Vortices in Helium ii. Cambridge Univ. Press, Cambridge

Espinoza C. M., Lyne A. G., Stappers B. W., Kramer M., 2011, MNRAS, 414, 1679

Fattoyev F., Piekarewicz J., 2010, Phys. Rev. C, 82, 025810

Haskell B., Melatos A., 2015, Int. J. Mod. Phys. D, 24, 1530008

Heinke C. O, Ho W. C. G., 2010, ApJ, 719, 167

Howitt G., Haskell B., Melatos A., 2016, MNRAS, 460, 1201

Jou D., Mongiovì M. S., 2004, Phys. Rev. B, 69, 094513

Jou D., Mongiovì M. S., Sciacca M., 2011, Physica D: Nonlinear Phenomena, 240, 249

Khalatnikov I., 1965, An Introduction to the Theory of Superfluidity, No. $532.5 \mathrm{~K} 5$
Kozik E., Svistunov B., 2009, J. Low Temp. Phys., 156, 215

Melatos A., Peralta C., 2007, ApJ, 662, L99

Middleditch J., Marshall F. E., Wang Q. D., Gotthelf E. V., Zhang W., 2006, ApJ, 652, 1531

Migdal A. B., 1959, Nucl. Phys., 13, 655

Mongiovì M. S., Jou D., 2007, in Das M., ed., Condensed Matter: New Research. Nova Science Publishers, New York, p. 1

Mongiovì M. S., Sciacca M., 2010, in Greco A., Rionero S., Ruggeri T., eds, Waves and Stability in Continuous Media. World Scientific, Singapore, p. 297

Nemirovskii S. K., 2013, Phys. Rep., 524, 85

Nemirovskii S. K., Stamm G., Fiszdon W., 1993, Phys. Rev. B, 48, 7338

Packard R. E., 1972, Phys. Rev. Lett., 28, 1080

Padmanabhan T., 2001, Theoretical Astrophysics. Vol. II: Stars and Stellar Systems. Cambridge Univ. Press, Cambridge

Page D., Prakash M., Lattimer J. M., Steiner A., 2011, Phys. Rev. Lett., 106, 081101

Peralta C., Melatos A., Giacobello M., Ooi A., 2006, ApJ, 651, 1079

Peralta C., Melatos A., Giacobello M., Ooi A., 2008, J. Fluid Mechanics, 609,221

Saluto L., Jou D., Mongiovì M. S., 2014a, Physica A, 406, 272

Saluto L., Jou D., Mongiovì M. S., 2014b, Physica B, 440, 99

Sciacca M., 2010, Mathematical and Computer Modelling, 51, 91

Sciacca M., Mongiovì M. S., Jou D., 2008, Physica B: Condensed Matter, 403, 2215

Sciacca M., Sergeev Y. A., Barenghi C. F., Skrbek L., 2010, Phys. Rev. B, 82,134531

Sedrakian D., Hayrapetyan M., Sedrakian L., 2007, Astrophysics, 50, 144

Sedrakian D., Hayrapetyan M., Baghdasaryan D., 2014, Astrophysics, 57,90

Shternin P. S., Yakovlev D. G., Heinke C. O., Ho W. C. G., Patnaude D. J., 2011, MNRAS, 412, L108

Swanson C. E., Barenghi C. F., Donnelly R., 1983, Phys. Rev. Lett., 50, 190

Tsakadze J., Tsakadze S., 1980, J. Low Temp. Phys., 39, 649

Urama J., Joshi B., Chukwude A., 2012, in Proc. IAU Symp. Vol. 291, Neutron Stars and Pulsars: Challenges and Opportunities after 80 years. Kluwer, Dordrecht, p. 533

van Eysden C. A., Melatos A., 2010, MNRAS, 409, 1253

Vinen W. F., 1957a, Proceedings of the Royal Society of London A, 240,114

Vinen W. F., 1957b, Proceedings of the Royal Society of London A, 240,128

Vinen W. F., 1957c, Proceedings of the Royal Society of London A, 240,493

Vinen W. F., 2001, Phys. Rev. B, 64, 134520

Wang N., Manchester R. N., Pace R. T., Bailes M., Kaspi V. M., Stappers B. W., Lyne A. G, 2000, MNRAS, 317, 843

Yu M. et al., 2013, MNRAS, 429, 688

\section{APPENDIX A: OUTLINE OF ROTATING SUPERFLUID TURBULENCE}

We briefly recall some results on the turbulence in rotating superfluid helium, which have inspired the construction of our mathematical model for glitches in pulsars.

It is well known that when one applies a rotation to a sample filled with superfluid helium, a vortex array (aligned to the rotation axis) is formed. A disordered tangle of quantized vortex lines is created in the so-called counterflow experiments (a channel filled with superfluid helium and heated at one end), when the heat flow is greater than a critical value. Using the two-fluid model, only the normal fluid component carries entropy and heat flow, so its motion will be from the heated end to the opposite end. At the same time, there is conservation of mass and so the superfluid component must counterflow towards the heated end. Thus, a relative counterflow is established between the normal fluid and superfluid components, 
characterized by the absolute value of the counterflow velocity $V=$ $\left\langle\left|\boldsymbol{v}_{\mathrm{n}}-\boldsymbol{v}_{\mathrm{s}}\right|\right\rangle$, where $\boldsymbol{v}_{\mathrm{n}}$ and $\boldsymbol{v}_{\mathrm{s}}$ denote the velocities of the normal and superfluid components, respectively.

In both cases (rotation only and counterflow only), the vortex array is described in terms of a macroscopic average of the vortex line length per unit volume $L$ (called the vortex line density, which has dimensions length ${ }^{-2}$ ).

In the steady state in counterflow experiments, the vortex line density $L$ is described well by

$L=\gamma_{\mathrm{H}} V^{2}$,

where $\gamma_{\mathrm{H}}$ is a coefficient dependent on the temperature. We refer to Donnelly (1991) for details.

In a rotating cylindrical sample, the line density $L$ (i.e. the number of vortex lines per unit surface, in this case) is given by

$L=\frac{2 \Omega}{\kappa}$,

where $\Omega$ denotes the modulus of the angular velocity and $\kappa$ is the quantum of vorticity (i.e. $\kappa=h / m_{4} \simeq 9.97 \times 10^{-4} \mathrm{~cm}^{2}$ $\mathrm{s}^{-1}$, involving the Planck constant $h$ and the mass $m_{4}$ of the ${ }^{4}$ He atom).

Historically, the first form of an evolution equation for $L$ under constant values of the counterflow velocity $V$ appears in Vinen $(1957 \mathrm{c})$ as

$\frac{\mathrm{d} L}{\mathrm{~d} t}=\alpha_{v} V L^{3 / 2}-\beta_{v} \kappa L^{2}$,

where $\alpha_{v}$ and $\beta_{v}$ are dimensionless constants. This equation was formulated by Vinen (1957a,b,c). Indeed, following the suggestion of Feynman - see also Barenghi et al. (2001) and Swanson et al. (1983) - Vinen assumes that the time derivative $\mathrm{d} L / \mathrm{d} t$ is composed of two terms:

$\frac{\mathrm{d} L}{\mathrm{~d} t}=\left(\frac{\mathrm{d} L}{\mathrm{~d} t}\right)_{\text {formation }}-\left(\frac{\mathrm{d} L}{\mathrm{~d} t}\right)_{\text {destruction }}$.

The first is responsible for the growth of $L$ and the second for its decay. The form of the destruction term was obtained in analogy with classical turbulence

$$
\left(\frac{\mathrm{d} L}{\mathrm{~d} t}\right)_{\text {destruction }}=-\beta_{v} \kappa L^{2}
$$

while the form of the formation term was supposed to be dependent on the quantum of circulation $\kappa$, on the instantaneous value of $L$ and on the force between the vortex line and the normal component (which is linked to the intensity of the counterflow velocity). The dimensional analysis has also been discussed by Vinen (1957a,b,c) and leads to

$$
\left(\frac{\mathrm{d} L}{\mathrm{~d} t}\right)_{\text {formation }}=\kappa L^{2} \phi_{\mathrm{f}}\left(\frac{V}{\kappa L^{1 / 2}}\right)
$$

where $\phi_{\mathrm{f}}$ is a suitable dimensionless function.

In the steady state, the combined rotation and the axial counterflow exhibit a rich set of features. A well-known experiment on simultaneous rotation and counterflow in superfluid helium is the apparatus of Swanson et al. (1983).
Experimental observations can be summarized as follows. A container, filled with superfluid helium, is rotating at a given angular speed $\Omega$ and an increasing heat flow (corresponding to a counterflow velocity $V$ ) is imposed. The presence of a critical counterflow rotation velocity $V_{\mathrm{C}}$ is found, which scales as $\Omega^{1 / 2}$. More precisely, for $V \leq V_{\mathrm{C}}$, the length $L$ per unit volume of the vortex lines is approximately independent of $V$ and proportional to the angular speed $\Omega$, so that $L=2 \Omega / \kappa$. However, for $V \geq V_{\mathrm{C}}, L$ depends also on $V$. The transition appears to be towards turbulence, when the ordered array begins to behave like a turbulent tangle. The tangle appears to be 'polarized' to accomplish the rotation. The effective polarization increases with rotation.

Jou \& Mongiovì (2004) studied an evolution equation for $L$ in the presence of counterflow and rotation. The destruction term, following Vinen, was modelled by equation (A5). The production term was assumed to be dependent on $V$ and $\Omega$, as well as on $\kappa$ and $L$. Dimensional analysis leads to

$$
\left(\frac{\mathrm{d} L}{\mathrm{~d} t}\right)_{\text {formation }}=\kappa L^{2} \phi_{\mathrm{f}}\left[\left(\frac{\Omega}{\kappa L}\right)^{1 / 2},\left(\frac{V}{\kappa L^{1 / 2}}\right)\right] .
$$

Therefore, the following generalization of equation (A3) was proposed,

$\frac{\mathrm{d} L}{\mathrm{~d} t}=-\beta \kappa L^{2}+\left(\alpha_{1} V+\alpha_{2} \sqrt{\kappa \Omega}\right) L^{3 / 2}-\left(\beta_{1} \Omega+\beta_{2} \frac{V \sqrt{\Omega}}{\sqrt{\kappa}}\right) L$,

with suitable dimensionless constants $\alpha_{1}, \alpha_{2}, \beta_{1}$ and $\beta_{2}$, which have to be determined by comparison with experimental data. The term in $V^{2}$ has been omitted in equation (A8), because the values of $V$ considered are not too high.

Sciacca et al. (2008) suggested another possible form for the evolution of $L$ in the presence of counterflow and rotation. This was a further generalization of the ideas of Vinen (1957a,b,c) and described in a better way some of the experimental results, assuming a different expression for the production terms. The expression of one of the main equations of evolution in Sciacca et al. (2008) is

$\frac{\mathrm{d} L}{\mathrm{~d} t}=-\beta \kappa L^{2}+A_{1}\left(L-v_{1} \frac{\Omega}{\kappa}\right) \frac{V^{2}}{\kappa}+B_{1}\left(L-v_{2} \frac{\Omega}{\kappa}\right) \Omega$,

where $A_{1}, B_{1}, v_{1}$ and $v_{2}$ are dimensionless constants.

With suitable values of the coefficients, both equations (A8) and (A9) are able to describe experimental results at various angular velocities. More details can be found in Mongiovì \& Jou (2007), Jou \& Mongiovì (2004), Sciacca et al. (2008) and Sciacca (2010).

This paper has been typeset from a $\mathrm{TE}_{\mathrm{E}} \mathrm{X} / \mathrm{L} \mathrm{T} \mathrm{E}$ file prepared by the author. 